\title{
Revisión metodológica del diseño de espaldones en diques de escolleras
}

\author{
Methodological review for the design of crown walls in rubble mound breakwaters
}

\section{Dalila Ruiz, Edgar Mendoza y Rodolfo Silva}

Fecha de entrega: 30 de mayo 2012 Fecha de aceptación: 24 de abril 2013

Coordinación de Hidráulica, Instituto de Ingeniería, Universidad Nacional Autónoma de México, Cd. Universitaria, 04510 D.F., México, DruizC@iingen.unam.mx, EmendozaB@iingen.unam.mx, RsilvaC@iingen.unam.mx

La utilidad de un dique con espaldón va más allá de una estructura que ofrece protección costera, sus alcances pueden abarcar también aspectos económicos, ecológicos y sociales. Los estudios cientificos existentes respecto de su diseño y funcionamiento son escasos y con ciertas limitantes de precisión. Por ello se debe tener una idea muy clara de las condiciones de aplicación en la selección de la metodología que mejor se adapte para lograr un diseño seguro y funcional. En este artículo se presenta una revisión del estado del arte sobre el diseño de estructuras de piezas sueltas coronadas con un espaldón impermeable y una comparación entre los diferentes métodos disponibles, para brindar a los proyectistas un panorama general de las ventajas y desventajas que ofrece cada uno. Se utilizan, como ejemplo, las condiciones de oleaje frente a una terminal de gas licuado en las costas de Baja California, México, donde es necesario generar una condición de calma para garantizar la seguridad del buque durante las maniobras de atraque y descarga del producto; ello requiere poca transmisión y rebase nulo, condiciones que un dique con espaldón es capaz de ofrecer de manera adecuada.

Palabras clave: espaldón, protección de costas, sub-presión
The purpose of a crown wall on a rubble mound breakwater goes further than coastal protection; it also offers economic, ecological and social advantages. Few scientific studies are available on design and performance of crown walls and they often have limited accuracy. So a clear understanding of the applicability conditions for the methodology selection that better suits a safe and functional design is needed This article presents a review of the state of the art of studies on crown wall structures and a comparison between different methods available to provide the designers with an overview of the advantages and disadvantages offered by each one. As an example, the wave conditions in front of a liquefied gas terminal, off the coast of Baja California, México, are used, where calm conditions are needed to ensure the security of the vessel during docking and unloading; this means low transmission and null overtopping, which makes crown wall breakwater a good option.

Keywords: crown wall, shore protection, uplift pressure

\section{Introducción}

La demanda mundial de bienes y productos ha tenido un crecimiento significativo en las últimas décadas. Las compañías navieras, en conjunto con las autoridades portuarias de cada región, han determinado el aumento de las dimensiones de las embarcaciones como resultado del incremento en las necesidades de transporte de mercancías. Estos buques de gran calado, a su vez, han obligado a los proyectistas a ampliar y mejorar las instalaciones portuarias y con ellas las estructuras de abrigo. De manera general se tiene que la cota de desplante de las obras de abrigo es cada vez más profunda y por ello las estructuras 
quedan expuestas a mayores solicitaciones al encontrarse con oleaje más energético.

En este escenario, el reto para la ingeniería marítimoportuaria radica en seleccionar la tipología que mejor cumpla con las necesidades de cada sitio y proyecto, optimizando costos y proponiendo estrategias para que el proceso constructivo sea asequible en estas nuevas profundidades.

En este sentido, el presente trabajo revisa y propone el uso de una tipología de estructura de piezas sueltas con superestructura rígida e impermeable, capaz de brindar un estado de servicio óptimo y seguro a las embarcaciones dentro del complejo portuario; este tipo de diques se conoce como dique con espaldón.

La piezas que conforman la parte baja de esta tipología trabajan por peso y trabazón, y la corona suele ser una pieza de concreto monolítica que impide el rebase en condiciones de tormenta (Figura 1).

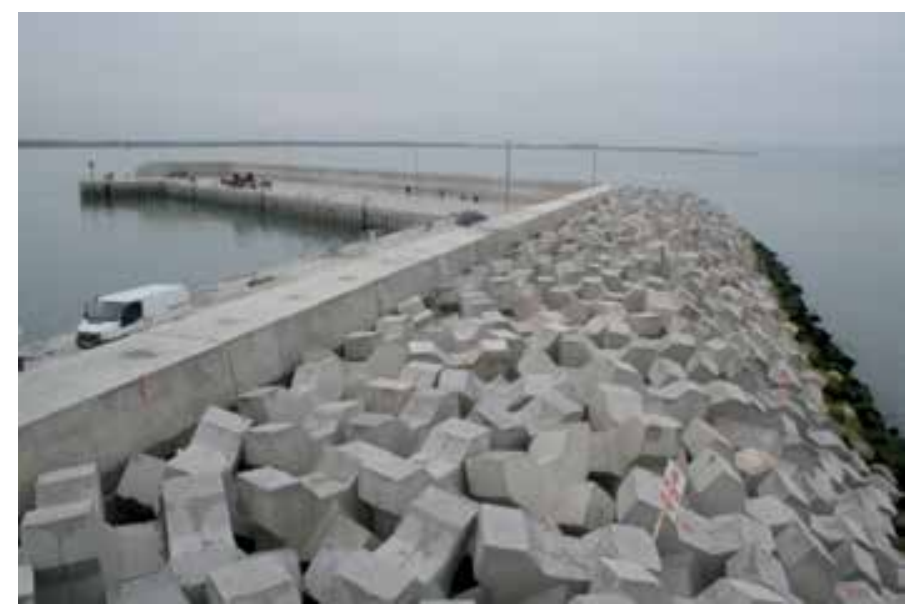

Figura 1: Dique con espaldón en Oriel, Irlanda

El funcionamiento hidráulico de un dique con espaldón, y por tanto su geometría, debe cumplir con la condición de rotura sobre el talud y evitar el impacto directo de las olas contra la pared vertical, es decir, la superestructura únicamente debe resistir los esfuerzos producidos por la lámina de agua en ascenso. En caso contrario, el dique debería considerarse como un dique mixto y su diseño se hará con las herramientas específicas de tipología mixta (e.g. Nagai, 1973; Takahashi et al., 2002) y no como espaldón.

Las ventajas que incentivan el uso de los espaldones son varias: como se mencionó antes, evitan el rebase del oleaje brindando un ambiente de calma a la navegación y atraque de buques. También mitigan los costos de acarreo de material para la construcción del dique, al sustituir parte del volumen de roca por una superestructura de concreto. Si se toma en cuenta que el costo de la roca es cada vez más elevado debido, entre otras, a la actual escasez de cantera o a su limitada explotación debido a regulaciones ecológicas, este factor adquiere relevancia.

Otra ventaja de este tipo de estructuras es el fácil acceso que se tiene al dique sobre el propio espaldón, que puede funcionar como vía de entrada ya sea para ofrecer servicios portuarios (e.g. cualquier tipo de reparación) o para fines recreativos como paseo marítimo o ciclo-vía. Así mismo, ofrecen la posibilidad de dotar de un muelle de atraque en la cara de sotamar del espaldón, lo cual puede ser de gran utilidad. A la vez, sobre el espaldón se pueden tender estructuras de carga/descarga de buques como cintas transportadoras y tuberías.

Algunos ejemplos de puertos mundiales donde se ha implementado el uso de diques con espaldón son: Puerto de Limbe en Camerún (www.dmc.nl/activities/coastalengineering/limbe-breakwater/item90), Puerto Oriel en Irlanda (www.xbloc.com/projects/breakwater-portoriel-ireland), Puerto Civitavecchia en Italia (www.portof-rome.org/), Puerto de Zeebrugge en Bélgica (www. portofzeebrugge.be/) y Puerto de Gijón en España (www. puertogijon.es/).

A la luz de lo anterior, el objetivo del presente trabajo es hacer una revisión de las metodologías de cálculo existentes para el diseño de diques con espaldón (i.e. estimación de las fuerzas que solicitan a la estructura de concreto) y llevar a cabo una comparación entre los resultados que ofrece cada una. Si bien, en la literatura existe un número reducido de métodos de cálculo, también se hace una recopilación de los estudios más recientes respecto de esta tipología.

\section{Método de Iribarren y Nogales}

Iribarren y Nogales (1964) propusieron un método prácticamente gráfico para determinar las presiones horizontales a las que está sometido un espaldón. La Figura 2 muestra un esquema de la relación de distancias que tomaron en cuenta estos autores para la estimación de dichas presiones. 


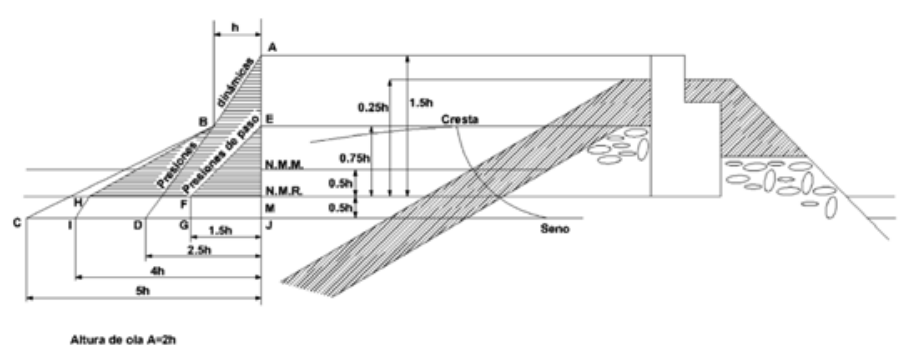

Figura 2: Esquema de presiones de una ola rota en un dique con espaldón, Iribarren y Nogales (1964)

Para entender la Figura 2 es necesario definir la nomenclatura de Iribarren y Nogales (1964), que es ligeramente distinta a la que utiliza el resto de autores, los autores denominan $A$ a la altura de ola, $h$ a la amplitud de ola y $H$ al calado. Estos autores especifican que el cálculo del espaldón es aproximado y definen $H=h$, por lo que la máxima velocidad horizontal del agua en la corona del enrocamiento queda definida como:

$$
V_{h}=C=\sqrt{g h}
$$

La altura representativa de la presión a la altura de la corona del enrocamiento es:

$$
E B=2 \frac{V_{h}^{2}}{2 g}=h
$$

si, por otro lado, la velocidad vertical de las partículas se define como:

$$
V_{v}=\sqrt{2 g 2 h}
$$

la velocidad resultante en la parte baja del espaldón es:

$$
V=\sqrt{V_{h}^{2}+V_{v}^{2}}=\sqrt{g h+4 g h}=\sqrt{5 g h}
$$

La altura del triángulo de presiones cuando ocurre el valle de la ola es:

$J C=2 \frac{V^{2}}{2 g}=5 h$

De acuerdo con Iribarren y Nogales (1964) la escollera reduce la presión a la mitad, por lo que la presión queda definida por la recta $A B D$ de la Figura 2 y, por reflexión instantánea en el espaldón vertical, la distancia $A M$ es igual a $1.5 A$, así, la ley total de presiones queda como la línea $A B H I$. Para Iribarren y Nogales (1964), el espaldón suele cimentarse al nivel medio del mar, por lo que las presiones ejercidas sobre la estructura quedan definidas por la línea $A B H$. Además de las presiones, se debe considerar el empuje de la escollera en ambas caras del espaldón, cuya estabilidad debe comprobarse ante los empujes totales al vuelco y deslizamiento.

Los espaldones fueron conocidos y construidos durante décadas, sin embargo, Iribarren y Nogales (1964) fueron de los primeros investigadores que observaron a detalle el desempeño de este tipo de estructuras y desarrollaron una metodología para su diseño. Sin embargo, a pesar del conocimiento que ellos generaron que, dicho sea de paso, es la base de muchos otros estudios, el método descrito posee limitaciones que hoy en día lo ponen fuera de competencia dado el elevado nivel de error que puede inducir su uso.

La principal desventaja es que considera que las presiones dinámicas (debidas al impacto de las olas) y las pseudohidrostáticas (debidas al ascenso) ocurren de manera simultánea, lo que lleva a que el cálculo sub-estime la fuerza horizontal cuando gobiernan las primeras y sobreestime cuando lo hacen las segundas. Iribarren y Nogales (1964) también proponen que el empuje generado por el valle de la ola equivale 5 veces a la amplitud de ola, lo cual no necesariamente ocurre ya que la roca del talud modifica la distribución de las presiones horizontales. Por último, la subpresión (empuje vertical en la base del espaldón) no es considerada dentro de los cálculos de este método.

Con todo lo anterior, la ventaja del modelo es su fácil aplicación, por lo que se recomienda solo para estimar, de manera preliminar, los empujes a los cuales es sometida una estructura.

\section{Modelo de Günbak y Gökce}

Este modelo (Günbak y Gökce, 1984) considera que la lámina de agua en ascenso forma un triángulo con ángulo de $15^{\circ}$ respecto del talud de roca (Figura 3 ), de donde se obtiene una elevación $y$, que representa la altura máxima del agua si el talud fuera infinitamente largo. 

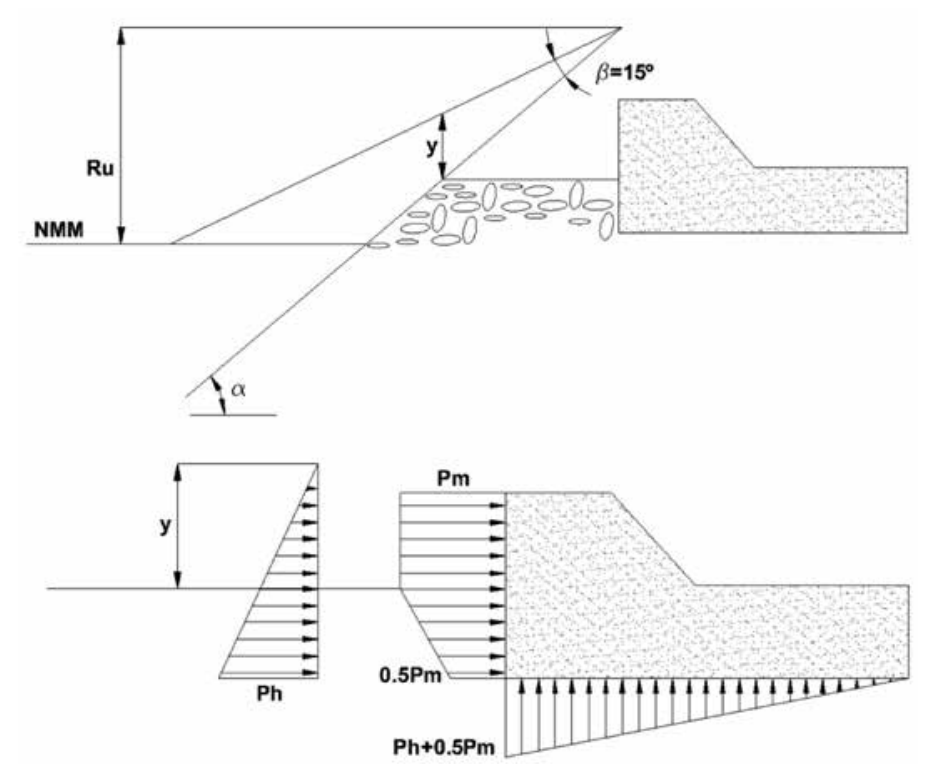

Figura 3: Esquema de Günbak y Gökce

La Figura 3 muestra la distribución de presiones horizontales propuesta por Günbak y Gökce (1984); los autores consideran $P_{m}$ como presión de choque y $P_{h}$ como presión cuasi-hidrostática, las cuales se pueden estimar como:

$$
\begin{aligned}
& P_{m}=\frac{\rho g(\sqrt{g y})^{2}}{2 g}=1.03 \frac{y}{2} \\
& P_{h}=\rho g(y+c-z) \\
& y=\frac{(R u-c)}{\operatorname{sen} \alpha} \frac{\operatorname{sen} \beta}{\cos (\alpha-\beta)}
\end{aligned}
$$

Donde $c$ es la cota de la berma que protege al espaldón respecto del nivel medio del mar, $\beta$ es el ángulo formado por la lámina de agua en ascenso, $\alpha$ es el ángulo del talud del dique y $z$ es la cota respecto del nivel medio del mar del punto donde se desea realizar el cálculo.

$R u$ es el ascenso máximo de la lámina de agua que se calcula con las siguientes expresiones:

$$
\begin{aligned}
& R u=0.4 \varepsilon H \text { si } \varepsilon<2.5 \\
& R u=H \text { si } \varepsilon>2.5
\end{aligned}
$$

donde

$$
\varepsilon=\sqrt{\frac{g}{2 \pi H}} T \tan \alpha
$$

Para la estimación de la subpresión se utiliza una ley triangular con valor $P_{h}+0.5 P_{m}$ en el extremo expuesto al oleaje y cero en el extremo protegido de la estructura (Günbak y Gökce, 1984).

Este método precisa que existan dos distribuciones diferentes de presiones horizontales, la de choque (dinámica) y la cuasi-hidrostática, las cuales considera de acción simultánea. Günbak y Gökce (1984) consideran una reducción del $50 \%$ en la presión dinámica actuante debido a la escollera, sin embargo, no presentan el fundamento para fijar tal porcentaje de reducción. Al ser un método con mayor sustento en la investigación, toma en cuenta las presiones en sentido vertical, que actúan en la base del espaldón como la suma de la presión horizontal tanto dinámica como cuasi-hidrostática, lo cual a la luz del conocimiento actual, en el que se sabe que dichas fuerzas no son simultáneas, tiende a sobre-estimar las fuerzas aplicadas.

La ventaja del método es que facilita la determinación de los valores de las variables para el cálculo de las presiones, sin embargo, aún queda cierta incertidumbre respecto de la estimación de las presiones verticales ya que la hipótesis de ley triangular no es necesariamente certera.

\section{Modelo de Pedersen y Burcharth}

El modelo de Pedersen y Burcharth (1992) se deriva de una serie de ensayos enfocados en obtener las fuerzas que actúan sobre un espaldón con dique de escollera. Estos autores estudiaron el efecto de distintos parámetros de forma experimental como la longitud de onda, altura de ola significante, altura de la berma de escollera, ancho de la berma de escollera y altura del espaldón.

De los ensayos realizados, Pedersen y Burcharth (1992) observaron que el ancho de la berma tiene poca influencia en las fuerzas finales actuantes, que la variación de las fuerzas se relaciona linealmente con la altura de ola significante y que la longitud de onda es un factor fundamental para la estimación de las fuerzas resultantes.

Los ensayos fueron realizados con oleaje irregular, por lo que los resultados obtenidos y el modelo de cálculo de las fuerzas están asociados a un estudio estadístico de probabilidad de ocurrencia de las fuerzas aplicadas. 
Los autores recomiendan, para diseño, la fuerza con probabilidad de ocurrencia de $0.1 \%$ (Figura 4 ).

$$
\frac{F_{0.1 \%}}{\rho g h_{f} L_{p}}=a+b\left(\frac{H_{s}}{A_{c}}\right)
$$

Donde $\rho g$ es el peso específico del agua, $h_{f}$ la altura total del espaldón, $L_{p}$ la longitud de onda máxima del espectro, $H_{s}$ la altura de ola significante y $A_{c}$ la altura de la berma medida desde el nivel medio del mar. Los parámetros $a$ y $b$ obedecen a los resultados experimentales que se muestran en la Figura 4.

El método propuesto no calcula las subpresiones, además, define una fuerza con probabilidad de $0.1 \%$ que no necesariamente responde a las necesidades de servicio últimos y límites de una estructura.

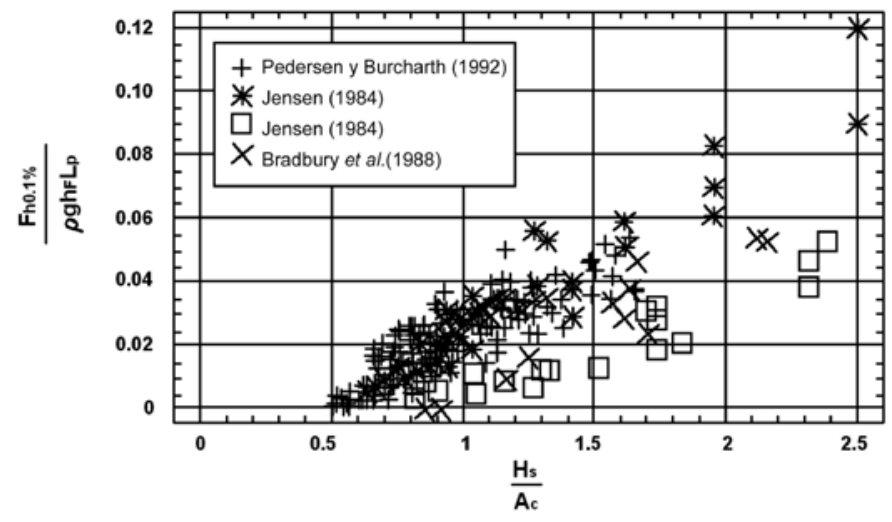

Figura 4: Datos experimentales de Pedersen y Burcharth (1992), Jensen (1984) y Bradbury et al. (1988)

Como este modelo toma sus bases del trabajo de Jensen (1984), los valores de $a$ y $b$ propuestos por dicho autor son válidos. La Tabla 1 muestra los valores de Jensen (1984) y los añadidos más tarde por Bradbury et al. (1988).

Tabla 1: Valores de los coeficientes $a$ y $b$ para la ecuación (12)

\begin{tabular}{|c|c|c|c|c|c|c|}
\hline \multirow{2}{*}{ Autor } & \multirow{2}{*}{ Sec. } & \multicolumn{4}{|c|}{$\begin{array}{c}\text { Rango de parámetros para las } \\
\text { pruebas }\end{array}$} & \multicolumn{2}{|c|}{$\begin{array}{c}\text { Valores de } a \text { y } b \\
\text { probabilidad de } \\
\text { excedencia } 0.1 \%\end{array}$} \\
\cline { 3 - 7 } & & $\mathrm{Ac}, \mathrm{m}$ & $\mathrm{S}_{\mathrm{op}}=\mathrm{H}_{\mathrm{s}} / \mathrm{L}_{\mathrm{op}}$ & $\mathrm{H}_{\mathrm{s}} / \mathrm{A}_{\mathrm{c}}$ & $a$ & $b$ \\
\hline Jensen & $\mathrm{A}$ & $5.6-10.6$ & $0.016-0.036$ & $0.76-2.5$ & -0.026 & 0.051 \\
\hline Jensen & $\mathrm{B}$ & $1.5-3.0$ & $0.005-0.011$ & $0.82-2.4$ & -0.016 & 0.025 \\
\hline Bradbury & $\mathrm{C}$ & 0.10 & $0.023-0.07$ & $0.9-2.1$ & -0.038 & 0.043 \\
\hline Bradbury & $\mathrm{D}$ & 0.14 & $0.04-0.05$ & 1.43 & -0.0247 & 0.028 \\
\hline Bradbury & $\mathrm{E}$ & 0.18 & $0.04-0.05$ & 1.11 & -0.0095 & 0.0107 \\
\hline
\end{tabular}

\section{Modelo de Martin}

Este modelo (Martin et al., 1999) es aplicable en espaldones sobre dique de escollera donde las olas rompen sobre el talud o en casos donde la ola rompe antes de llegar al dique. El autor destaca la importancia de verificar que la ola no impacte directamente contra el espaldón para asegurar que las acciones sobre la estructura sean producidas por el fenómeno de asenso y descenso del agua sobre el talud.

Martin et al. (1999) plantean el cálculo por separado de las presiones dinámicas y pseudo-hidrostáticas al sostener que son originadas por distintos fenómenos y que no son simultáneas. La decisión sobre cuál se empleará en el diseño se toma con base en una comparación directa seleccionando la que genere menor estabilidad, lo cual depende de los coeficientes de seguridad que sean admitidos en cada localidad. Esta misma presión se emplea para estimar la subpresión.

El primer paso para el cálculo de presiones es conocer el valor del ascenso máximo bajo el supuesto de talud infinito; en función de éste es fácil determinar el ancho de la lámina de agua en la corona del talud de escollera como:

$$
S=H\left(1-\frac{A_{c}}{R_{u}}\right)
$$

donde $A_{c}$ es la cota de coronación de la escollera. La distribución de presiones dinámicas es constante entre $A_{c} \mathrm{y}$ $A_{c}+S$ con un valor igual a $\alpha \rho g S$, donde $\alpha$ es función del ascenso máximo y del ángulo del talud del dique respecto del fondo, por lo que contiene la información de la celeridad de aproximación de la lámina de agua de ancho $S$ (Martin et al., 1999). $\alpha$ se define por la siguiente expresión:

$$
\begin{aligned}
& \alpha=2 \frac{R_{u}}{H \cos ^{2} \beta \cos \theta} \\
& \alpha=2.9\left[\frac{R u}{H} \cos \beta\right]^{2}
\end{aligned}
$$

Donde el run-up se puede determinar, por ejemplo, a partir del método de Losada y Giménez-Curto (1981):

$$
\frac{R u}{H}=A u\left(1-e^{B u I r}\right)
$$


Para diques homogéneos:

$$
\begin{aligned}
& A u=-4.706 n+3.293 \\
& B u=-1.569 n+0.038
\end{aligned}
$$

La distribución de presiones en la zona protegida por la escollera debe ser menor debido a que el impacto es soportado por los elementos del talud. El porcentaje de presión en esta zona es función de múltiples parámetros como la porosidad, velocidad del flujo, ancho de la escollera, entre otros; por lo que resulta complicado determinarlo teóricamente. Por ello se admite un coeficiente de reducción $\lambda$ basado en lo propuesto por Günbak y Gökce (1984). Al desconocer la distribución de las presiones en la parte del espaldón cubierto por la escollera, se propone constante con valor igual a $\lambda \alpha \rho g S$ que actúa desde la cimentación del espaldón hasta $A_{c}$.

$$
\begin{aligned}
& \lambda=0.8 e^{-10.9 \frac{B}{L}} ; P d=\alpha \rho g S ; A_{c} \geq S \\
& \lambda P d=\lambda \alpha \rho g S ; \text { Cota de desplante } \geq A_{c}
\end{aligned}
$$

Para determinar la distribución de presiones horizontales, el autor recurre a ensayos experimentales que muestran una similitud entre la ley de presión pseudo-hidrostática y la hidrostática únicamente variando por un factor $\mu$ (Martin et al., 1999), quedando la distribución como:

$$
P_{h}(Z)=\mu \rho g\left(S+A_{c}-Z\right)
$$

Al calcular la subpresión, Martin et al. (1999) proponen una ley lineal entre los extremos (expuesto y protegido) del espaldón, donde el lado expuesto tendrá el valor máximo entre la presión pseudo-hidrostática y la presión dinámica reducida y en el extremo protegido el valor es nulo.

La Figura 5 muestra el diagrama de presiones según el autor y la región en la que actúa cada una de ellas. El método de Martin et al. (1999) se fundamenta en diversos trabajos que, al integrarse, forman una metodología más completa para el cálculo de presiones sobre un espaldón. Sin embargo, el cálculo de las presiones en sentido vertical aún es muy simplista y podría ser poco preciso aceptar una distribución lineal partiendo de un máximo en el extremo expuesto que disminuye hasta cero en el extremo protegido, aunado a que el valor de la máxima subpresión es el valor máximo de la comparación entre dos presiones horizontales. Adicionalmente, la hipótesis de que el valor máximo de la subpresión y el de la mayor fuerza horizontal ocurren de manera simultánea puede estar lejos de la realidad.
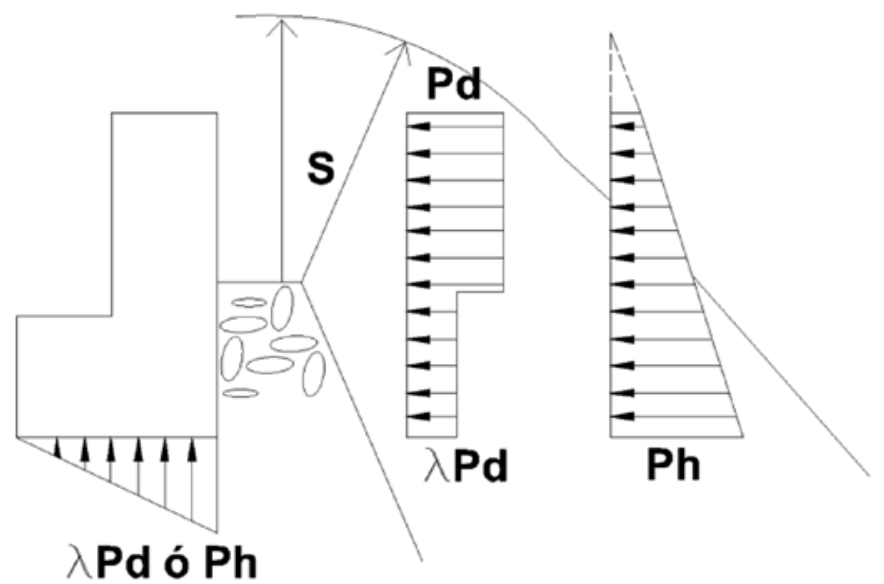

Figura 5: Diagrama de presiones (Martin et al., 1999)

\section{Modelo de Pedersen}

Pedersen (1996) desarrolló un modelo semi-empírico basado en los estudios de Günbak y Gökce (1984) combinado con un análisis paramétrico de los resultados de las pruebas del modelo físico. Este autor encontró que las fuerzas máximas son generadas únicamente por los impactos de olas. La formulación que dedujo tras los experimentos es la estimación, para un estado de mar determinado, de la fuerza horizontal, los momentos y la presión vertical con una probabilidad del $0.1 \%$ de excedencia:

$$
\begin{gathered}
F h_{0.1 \%}=0.21 \sqrt{\frac{L_{0 m}}{B}}\left(1.6 p_{m} y_{\text {eff }}+V \frac{p_{m}}{2} h_{p r o t}\right) \\
M_{0.1 \%}=0.55\left(h_{p r o t}+y_{e f f}\right) F h_{0.1 \%} \\
P b_{0.1 \%}=1 V p_{m}
\end{gathered}
$$

El ascenso máximo se determina mediante la siguiente ecuación:

$$
R u_{0.1 \%}=0.9 R u_{2 \%}
$$

La validez de los estudios de Pedersen se limita a los rangos mostrados en la Tabla 2 (Pedersen, 1996). 
Tabla 2: Rangos de aplicación del método de Pedersen (1996)

\begin{tabular}{|c|c|}
\hline$\xi_{\mathrm{m}}$ & $1.1-4.2$ \\
\hline $\mathrm{H}_{\mathrm{s}} / \mathrm{A}_{\mathrm{c}}$ & $0.5-1.5$ \\
\hline $\mathrm{R}_{\mathrm{c}} / \mathrm{A}_{\mathrm{c}}$ & $1.0-2.6$ \\
\hline $\mathrm{A}_{\mathrm{c}} / \mathrm{B}$ & $0.3-1.1$ \\
\hline Cota & $1.5-3.5$ \\
\hline
\end{tabular}

\section{Acciones del oleaje sobre espaldones y sus efectos de escala en el laboratorio}

Martin et al. (2000) se enfocaron en el estudio de las fuerzas horizontales, subpresión y efectos de escala sobre diques de escolleras con espaldón para desarrollar modelos y herramientas para el diseño de la superestructura estableciendo las condiciones de carga sobre el espaldón. Los resultados experimentales entregaron como resultado tres series de datos medidos sobre una misma geometría correspondiente al Dique Príncipe de Asturias en el puerto de Gijón. La primera serie corresponde a datos medidos sobre el dique real, la segunda corresponde a datos medidos en el laboratorio a escala 1:90 y la tercera a datos medidos en el laboratorio a escala 1:18.4.

Martin et al. (2000) encontraron que el método de Martin et al. (1999) para el cálculo de fuerzas horizontales en diques permeables tiene un ajuste semejante a los datos obtenidos del laboratorio, sin embargo, para el caso de diques impermeables debe hacerse una corrección del parámetro $\mu$.

Para la determinación de la subpresión, Martin et al. (2000) destacan que influyen distintos factores en la evolución de las presiones a lo largo de la estructura, a su vez, verificaron que en la parte posterior del espaldón es inadecuado asumir una presión nula. Su aportación más importante es un método para el diseño de ensayos en el laboratorio donde involucra los factores antes mencionados para evitar o disminuir los efectos de escala en los experimentos.

\section{Fuerzas sobre espaldones: evaluación de formulaciones empíricas}

El trabajo presentado por Camus y Guillén (2004) compara los resultados de los modelos hidráulicos de cuatro rompeolas en España, contra algunas formulaciones empíricas para el diseño de diques de escolleras con espaldón. La verificación de cada modelo individualmente no ha sido registrada, es por ello que los autores realizaron una guía útil para los diseñadores donde se muestran las fortalezas y debilidades de cada método considerando las características geométricas del dique y del oleaje.

En el Centro de Estudios de Puertos y Costas en Madrid, Camus y Guillén (2004) realizaron pruebas sobre 4 modelos de diques, los modelos fueron establecidos mediante la ley de Froude y el número de estabilidad. Las condiciones de oleaje fueron obtenidas por medio de un análisis de pasos descendentes por cero de la serie temporal de superficie libre que fue registrada por sensores de nivel. Las fuerzas actuantes sobre la estructura fueron determinadas a través de integración trapezoidal de los datos obtenidos de los sensores de presión.

La conclusión de mayor relevancia para estos autores es la consolidación de la formulación de Pedersen (1996) como la de mayor confianza para la estimación de la máxima fuerza horizontal, las fuerzas debidas a la subpresión y los momentos actuantes bajo un determinado estado de mar.

\section{Modelación numérica sobre espaldones de pared curva}

El modelo numérico que presentan en la Universidad de Dalian en China (Li et al., 2011), pretende simular el campo de velocidades y presiones que actúan frente a un espaldón de pared curva, así como el perfil de la superficie libre del agua. El trabajo consistió en utilizar un método de volumen de fluido (VOF por sus siglas en inglés) basado en un sistema de coordenadas de ajuste (BFC) que describe con gran precisión los límites de estructuras costeras complejas, como es el caso de un espaldón de pared curva (Figura 6).

La validación del modelo se llevó a cabo comparando los resultados numéricos contra los obtenidos por medio de trabajo experimental. El modelo BFC simula con mayor precisión la interacción entre el oleaje y la pared curva del espaldón, es decir, se obtiene una mejoría en la estimación del campo de flujo, el campo de presiones y los perfiles temporales de la superficie libre del agua.

El trabajo presentado por Li et al. (2011) no cuenta con una metodología de diseño ni una ecuación para la determinación de la distribución de presiones, sin embargo, los resultados arrojados por el modelo numérico son una 
herramienta práctica para la propuesta de diseño de un espaldón de pared curva al disminuir el tiempo requerido en pruebas de laboratorio.

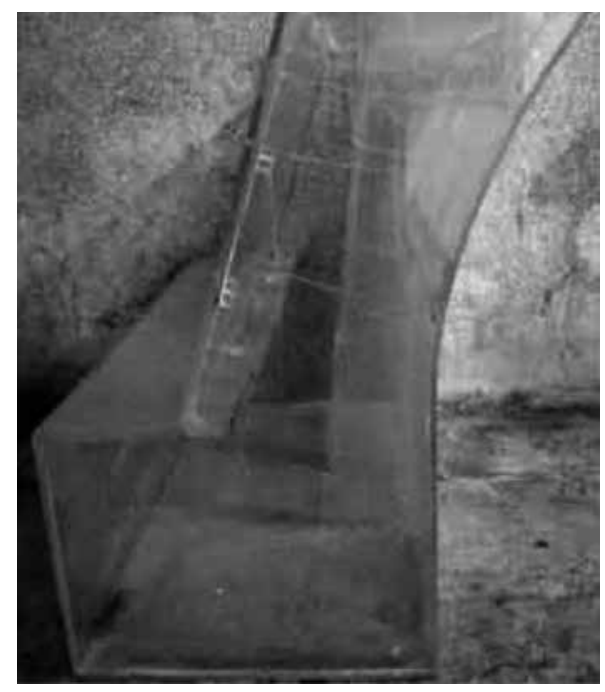

Figura 6: Modelo experimental de un espaldón de pared curva (Li et al., 2011)

\section{Diques berma con espaldón}

Tørum et al. (2012) presentaron un estudio experimental de las fuerzas actuantes sobre un espaldón con dique berma. Esta clasificación de diques es conocida por permitir el reacomodo de sus piezas con la dinámica marina, además de ser poco común su uso combinado con espaldones.

Los experimentos fueron realizados dentro de un canal de oleaje con un modelo de escolleras que permitiera reducir los factores de escala y un modelo del espaldón fabricado de aluminio (Tørum et al., 2012). Cinco sensores de presión fueron colocados en la pared vertical de aluminio y 3 sensores en la base del espaldón. La Figura 7 muestra un ejemplo de las presiones medidas en la pared vertical de la estructura y la Figura 8 muestra, para la misma condición de oleaje, la gráfica de presiones correspondientes a la subpresión.

Los resultados obtenidos muestran que las fuerzas a las cuales está sujeto un espaldón sobre un dique tipo berma son menores en comparación con las obtenidas en los diques de piezas sueltas (Tørum et al., 2012), que a su vez son los más estudiados. Se muestra una distribución de presiones no lineal, sin embargo, el estudio no deriva en una formulación que pueda ser aplicada para el diseño de espaldones.

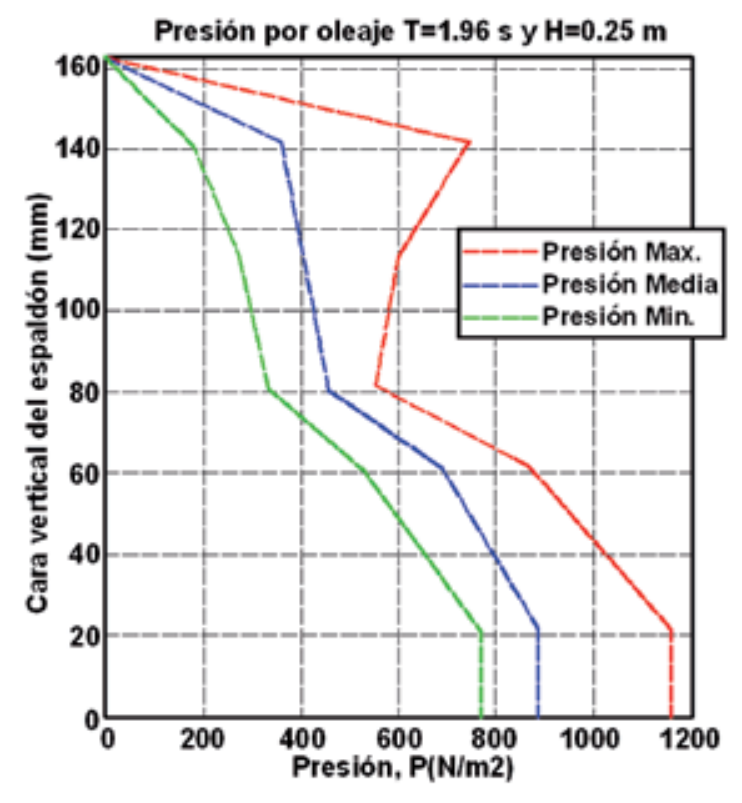

Figura 7: Distribución de presiones horizontales (tomado de Tørum et al., 2012)

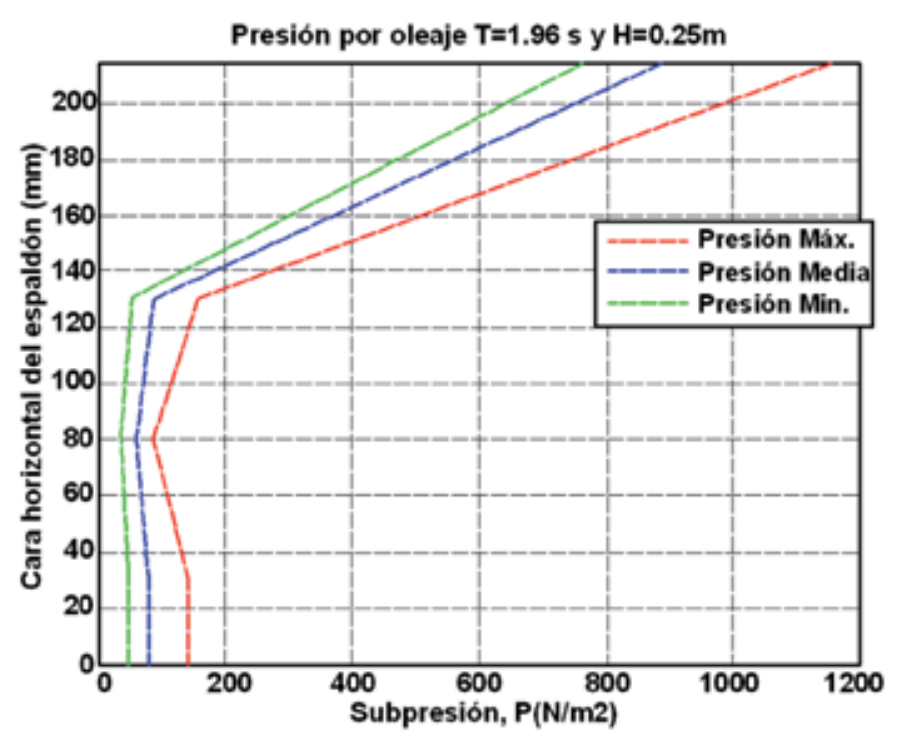

Figura 8: Distribución de presiones verticales (tomado de Tørum et al., 2012)

\section{Aplicación a un caso real}

Esta sección presenta una comparación entre los cuatro métodos disponibles para determinar las presiones a las cuales está sujeta la estructura y el coeficiente de seguridad por deslizamiento según la magnitud de los esfuerzos, con la finalidad de demostrar que los resultados son notablemente distintos, por lo que la selección del método 
de cálculo queda sujeto al criterio del proyectista y a los parámetros locales.

La ecuación para la estimación del coeficiente de seguridad se muestra a continuación, para la cual se han tomado valores de uso común en México y que pueden variar según la normatividad de cada país:

$$
C D=\frac{\left(\sum F y\right) 0.7}{\sum F h}>1.2
$$

donde $\sum F y$ es la sumatoria de fuerzas verticales y $\sum F h$ la de fuerzas horizontales.

Se tomará como ejemplo de cálculo las condiciones hidrodinámicas extremas frente a Salsipuedes, Baja California en México donde se propone un dique con espaldón para la protección de un muelle de descarga de gas. La selección del sitio para la ubicación del dique se basó en las necesidades de navegación de la empresa regasificadora (Figura 9). Un dique con espaldón, en este caso, tendría las ventajas de ofrecer condiciones óptimas para la navegación en la zona protegida, además de brindar un espacio de atraque para los buques utilizando como vía de acceso el espaldón; funcionando como muelle provisional durante los tiempos de espera o para mantenimiento de embarcaciones.

Se determinó una altura de ola significante de $6.3 \mathrm{~m}$ con un periodo de cálculo de $15.4 \mathrm{~s}$ que corresponden a condiciones hidrodinámicas para un periodo de retorno de 25 años en la zona de estudio.

El espaldón es una pieza de concreto con pared vertical que se cimienta en la cota $-2.0 \mathrm{~m}$, con una cota de coronación de $11.0 \mathrm{~m}$, cuyo peso específico es de $2300 \mathrm{~kg} / \mathrm{m}^{3}$. El manto conformado por roca que lo protege tiene una cota de cimentación de $-21.0 \mathrm{~m}$ y una cota de coronación de 6.0 $\mathrm{m}$, tomando como 0.0 el nivel medio del mar. El talud de la cara expuesta tiene una pendiente $2.5: 1$ y un ancho de berma de $6.4 \mathrm{~m}$, que corresponde a dos piezas de roca de 45 toneladas cada una. La Figura 10 muestra un esquema de la sección transversal del dique. Dado que el rango medio de marea local es cercano a $1.5 \mathrm{~m}$ no se considerará en los cálculos y por tanto el NMR se fijará al nivel medio del mar.

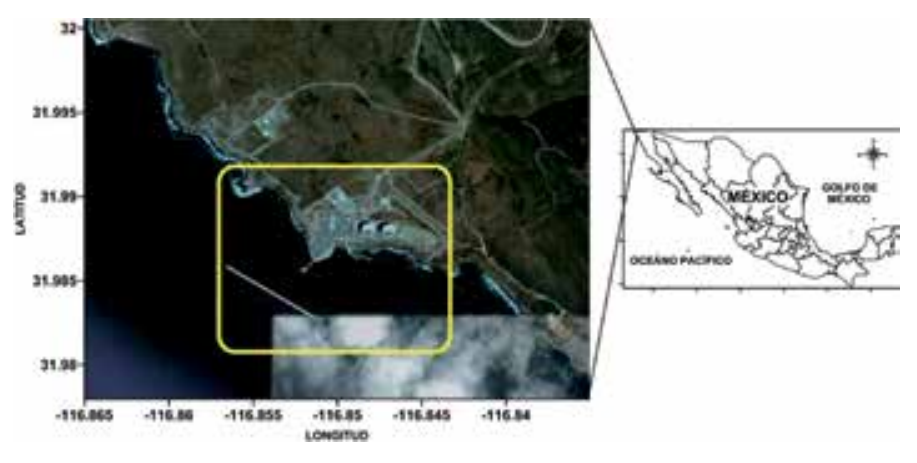

Figura 9: Mapa de ubicación

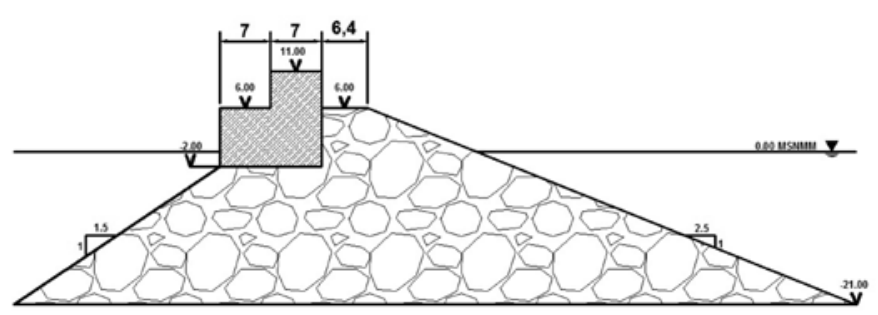

Figura 10: Sección tipo del dique

\section{Método de Iribarren}

En el modelo de Iribarren es necesaria la estimación de la línea $A B H I$ (Figura 1) por lo que se debe estimar para el cálculo del ascenso máximo del agua el valor de la altura de ola máxima $H_{\max }=1.6 H_{S}$. Así, la altura amplitud de ola para el método es $h=1.6 \cdot 6.3 / 2=5.04 \mathrm{~m}$. El ascenso máximo del agua (cota del punto A desde el NMR) es $\mathrm{s}=1.5 \mathrm{~A}=$ $3 h=3 \cdot 5.04=15.12 \mathrm{~m}$, dado que el NMR y la base del espaldón coinciden, la ley de presiones queda descrita por la línea $\mathrm{ABH}$. Para determinar el empuje correspondiente se calculará el área del triángulo $\mathrm{ABE}$ y la del trapecio $\mathrm{EBHM}$, así $\mathrm{ABE}=1.5 \mathrm{~h}^{2} \rho \mathrm{g} / 2=1.5 \cdot 5.04^{2} 1025 / 2=19527.48$ $\mathrm{kg} / \mathrm{m}$, considerando que las líneas IH y DB son paralelas la línea $\mathrm{MH}$ tiene por longitud 3.75h, así, el trapecio EBMH queda, $\mathrm{EBHM}=(\rho \mathrm{gh}+\rho \mathrm{g} 3.75 h) 1.5 h / 2=(1025 \cdot 5.04+$ $1025 \cdot 3.75 \cdot 5.04) 1.5 \cdot 5.04 / 2=92755.53 \mathrm{~kg} / \mathrm{m}$. La sumatoria de fuerzas horizontales es: $112283.01 \mathrm{~kg} / \mathrm{m}$. El método no considera subpresiones, por lo tanto no es posible definir un coeficiente de seguridad por deslizamiento. Además es de notar que el espaldón, según este método, estaría sujeto a rebase.

\section{Modelo de Günbak y Gökce}

La distribución de presiones en este método se determina 
por medio de los parámetros $P_{m}$ y $P_{h}$. El valor $y$ se obtiene con la ecuación (8), siendo necesario el cálculo del run-up sobre el talud, a partir del valor de la altura de ola máxima, siguiendo la ecuación (9) y (11):

$$
\varepsilon=\sqrt{\frac{g}{2 \pi H}} T \tan \alpha=\sqrt{\frac{9.81}{2 \pi 10.08}} 15.4 \cdot \frac{1}{2.5}=2.42
$$

Como $\varepsilon<2.5, \mathrm{Ru}=0.4 \varepsilon \mathrm{H}=0.4 \cdot 2.42 \cdot 10.08=9.76 \mathrm{~m}$. Como se explicó antes, el cálculo en este trabajo no considera la marea dado que el rango es bajo.

$$
y=\frac{R u-c}{\operatorname{sen} \alpha} \frac{\operatorname{sen} \beta}{\cos (\alpha-\beta)}=\frac{(9.76-6)}{\operatorname{sen}(21.8)} \frac{\operatorname{sen}(15)}{\operatorname{sen}(21.8-15)}=2.64 \mathrm{~m}
$$

La presión de choque $P m$ únicamente se calculará para determinar, por medio de triángulos semejantes, el valor de la presión actuante a la altura correspondiente al run-up $\left(P m_{R u}\right)$ y con ello obtener el área del polígono de presiones.

$$
P m=\rho g \frac{y}{2}=1025 \cdot \frac{2.64}{2}=1353 \mathrm{~kg} / \mathrm{m}^{2}
$$

La presión cuasi-hidrostática es:

$$
P h=\rho g(y+c-z)=1025(2.64+6-(-2))=10906 \mathrm{~kg} / \mathrm{m}^{2}
$$

la presión reducida por la acción de la escollera queda,

$0.5 \mathrm{Pm}=0.5 \cdot 1353=676.5 \mathrm{~kg} / \mathrm{m}^{2}$

y la subpresión es:

$P u=P h+0.5 P m=10906+676.5=11582.5 \mathrm{~kg} / \mathrm{m}^{2}$

Con ello, las fuerzas actuantes se definen con cuatro áreas:

1) la del rectángulo de lado $P m$ y de altura $y$,

$$
A_{1}=P m \quad y=1353 \cdot 2.64=3571.92 \mathrm{~kg} / \mathrm{m}
$$

2) la del trapecio con $P m$ y $0.5 P m$ como bases mayor y menor, respectivamente y altura $c-z$,

$$
\begin{aligned}
& A_{2}=\frac{1}{2}(P m+0.5 P m)(c-z)=0.5(1353+676.5) \\
& (2.64-(-2))=4708.44 \mathrm{~kg} / \mathrm{m}
\end{aligned}
$$

3) la del triángulo cuasi-hidrostático,

$$
\begin{aligned}
& A_{3}=\frac{1}{2} P h(y+c-z)=0.5 \cdot 10906(2.64+6-(2)) \\
& =58019.92 \mathrm{~kg} / \mathrm{m}
\end{aligned}
$$

4) la del triángulo que define la ley de subpresiones ( $d$ es el ancho del espaldón)

$$
\begin{aligned}
A_{4} & =\frac{1}{2}(P h+0.5 P m) d=0.5(10906+676.5) 14 \\
& =81077.5 \mathrm{~kg} / \mathrm{m}
\end{aligned}
$$

En resumen las fuerzas ejercidas sobre el espaldón son:

Horizontal: $3571.92+4708.44+58019.92=66300.28 \mathrm{~kg} / \mathrm{m}$

Vertical: $81077.5 \mathrm{~kg} / \mathrm{m}$

Para determinar el coeficiente de seguridad por deslizamiento se requiere conocer el peso del espaldón, a saber,

Peso $=\rho_{c} g A-\rho g A_{s}=2300(14 \cdot 13-5 \cdot 7)-1025$.

$(2 \cdot 14)=309400 \mathrm{~kg} / \mathrm{m}$

donde $\rho_{c}$ es la densidad del concreto y $A_{s}$ el área sumergida del espaldón. Con ello, el coeficiente de seguridad por deslizamiento queda definido por la ecuación (23):

$$
C D=\frac{\left(\sum F y\right) 0.7}{\sum F h}=\frac{0.7(309400-81077.5)}{66300.28}=2.41
$$

\section{Modelo de Pedersen-Burcharth}

En este método es correcta la utilización de la altura de ola significante para realizar los cálculos y los coeficientes $a$ y $b$, de acuerdo con la Tabla 1, dado que la sección de dique aquí estudiada corresponde con la sección A de dicha tabla, $a=-0.026$ y $b=0.051$. La fuerza queda determinada por la ecuación (12):

$$
\begin{aligned}
& F_{0.1 \%}=\rho g h_{f} L_{P}\left(a+b \frac{H s}{A c}\right)= \\
& =1025 \cdot 13 \cdot 207.86\left(-0.026+0.051 \frac{6.3}{6}\right)=76306.19 \mathrm{~kg} / \mathrm{m}
\end{aligned}
$$

Este método no calcula la subpresión, por lo tanto no se puede definir el coeficiente de seguridad por deslizamiento. 


\section{Modelo de Martin}

Se determina el run-up a partir de la ecuación (15) para poder calcular el ascenso máximo de la lámina de agua y el valor de $\alpha$. Se utiliza el valor de la altura de ola máxima $H_{\max }$ para realizar los cálculos. Considerando un dique de roca homogénea, $\mathrm{n}=0.4$ :

$$
\begin{aligned}
& A u=-4.706(0.4)+3.293=1.41 \\
& B u=-1.569(0.4)+0.038=-0.59 \\
& R u=1.41\left(1-e^{-0.59 * 1.81}\right) 10.08=9.33 \mathrm{~m}
\end{aligned}
$$

Se observa que el valor del run-up recién calculado y el hallado con el método de Günbak y Gökce (1984) son muy parecidos y ninguno alcanza el nivel suficiente para pronosticar rebase. Los parámetros del modelo de Martin et al. (1999) son:

$$
S=10.08\left(1-\frac{6}{9.33}\right)=3.60 \mathrm{~m}
$$

que muestra un metro de diferencia, hacia arriba, respecto del método de Günbak y Gökce (1984) a pesar que el run$u p$ de este último es un poco mayor $\mathrm{y}$

$$
\alpha=2.9\left[\frac{9.35}{10.08} \cos (15)\right]^{2}=2.33
$$

La distribución de presiones se determina a partir de $P d$, $\lambda P d$ y $P h$ :

$$
\begin{aligned}
& P d=2.33 \cdot 1025 \cdot 3.6=8605 \mathrm{~kg} / \mathrm{m}^{2} \\
& \lambda=0.8 e^{-10.9 \cdot \frac{6.4}{207.86}}=0.57 \\
& \lambda P d=0.57 \cdot 8605=4921.35 \mathrm{~kg} / \mathrm{m}^{2}
\end{aligned}
$$

$\mu=0.62$ para 2 piezas de roca en la berma,

$$
P h(-2)=0.62 \cdot 1025(3.6+6-(-2))=7377.5 \mathrm{~kg} / \mathrm{m}^{2}
$$

Ahora se tienen dos situaciones, el caso en que las fuerzas dinámicas solicitan al dique, donde las fuerzas se describen por las áreas de los rectángulos: 1) $P d$ de ancho y $S$ de alto, 2) $\lambda P d$ de ancho y $A c-z$ de alto y el triángulo de subpresión con base $P d$ y altura $d$ (el ancho del espaldón), a saber,

$$
\begin{aligned}
& A_{1}=P d S=8605 \cdot 3.6=30978 \mathrm{~kg} / \mathrm{m} \\
& A_{2}=\lambda P d(A c-z)=4921.35(6-(-2))=39370.8 \mathrm{~kg} / \mathrm{m} \\
& A_{3}=\frac{1}{2} \lambda P d d=0.5 \cdot 4921.35 \cdot 14=34449.45 \mathrm{~kg} / \mathrm{m}
\end{aligned}
$$

En resumen, las fuerzas aplicadas son:

Horizontales: $70348.8 \mathrm{~kg} / \mathrm{m}$

Vertical $=34449.45 \mathrm{~kg} / \mathrm{m}$

Con ello, el coeficiente de seguridad por deslizamiento es,

$$
C D=\frac{\left(\sum F y\right) 0.7}{\sum F h}=\frac{0.7(309400-34449.45)}{70348.8}=2.74
$$

La segunda situación es cuando la fuerza pseudohidrostática solicita al dique, en cuyo caso las fuerzas son las áreas de los triángulos de presión horizontal y vertical.

$$
\begin{aligned}
A_{1} & =\frac{1}{2} P h\left(S+A_{c}-z\right)=0.5 \cdot 7377.5(3.6+6-(-2)) \\
& =42789.5 \mathrm{~kg} / \mathrm{m} \\
A_{2} & =\frac{1}{2} P h d=0.5 \cdot 7377.5 \cdot 14=51642.5 \mathrm{~kg} / \mathrm{m}
\end{aligned}
$$

Ahora el coeficiente de seguridad por deslizamiento es:

$$
C D=\frac{\left(\sum F y\right) 0.7}{\sum F h}=\frac{0.7(309400-51642.5)}{42789.5}=4.22
$$

Dado que el coeficiente de seguridad por deslizamiento en el caso de fuerzas dinámicas es menor, es el que se utiliza para diseño y para la comparación objeto de este trabajo.

\section{Conclusiones}

En Latinoamérica existe poca demanda referente a diques con espaldón debido al escaso conocimiento de los beneficios que brindan este tipo de estructuras. Es importante, sin embargo, considerar las ventajas y desventajas de cada tipología de estructura para determinar el diseño más apropiado aún cuando determinada alternativa sea poco común. En cuanto a los modelos comparados, los resultados obtenidos muestran valores dentro de un rango 
razonable. La Tabla 3 resume los resultados más relevantes.

Tabla 3: Resumen de resultados

\begin{tabular}{|l|c|c|c|}
\hline Modelo & $\begin{array}{c}\text { F. Horizontal, } \\
\mathrm{kg} / \mathrm{m}\end{array}$ & Subpresión, $\mathrm{kg} / \mathrm{m}^{2}$ & C.D. \\
\hline Iribarren & 112283.01 & - & - \\
\hline G\&G & 66300.28 & 81077.50 & 2.41 \\
\hline P\&B & 76306.19 & - & - \\
\hline Martin & 70348.80 & 34449.45 & 2.74 \\
\hline
\end{tabular}

Se observa que, de cuatro modelos, únicamente dos consideran las presiones verticales, por lo tanto es posible calcular un coeficiente de seguridad por deslizamiento con mayor precisión como es el caso del modelo de GünbakGökce (1984) y Martin et al. (1999). Los cuales ofrecen valores de las fuerzas horizontales muy parecidos y pese a que el primero estima fuerzas verticales casi del doble de las que se obtienen con el segundo, el valor del coeficiente de seguridad por deslizamiento resulta muy parecido. Es de notar que los valores de ascenso máximo de ambos métodos son, también muy similares.

Desde un punto de vista ingenieril, el modelo Pedersen y Bucharth (1992) puede no ser práctico ya que define la fuerza con probabilidad $0.1 \%$, lo que podría no ajustarse a las necesidades reales de un sitio específico y no considera una distribución de presiones a lo largo del espaldón para realizar un diseño óptimo que se adecúe a los forzamientos del mar. En todo caso, dicha restricción explica claramente la diferencia en el valor de las fuerzas que se obtiene con esta metodología.

El modelo de Iribarren (1964) es un método sencillo que, aun cuando en la teoría reconoce dos tipos de presiones, en el cálculo las integra y esto lo lleva a sobreestimar las fuerzas.

Finalmente, se destacan dos puntos importantes que deben ser considerados por los proyectistas para prevenir daños en un dique con espaldón. Primero, se debe poner atención en la estimación del valor de la presión vertical, sin embargo, la sensibilidad del coeficiente de seguridad por deslizamiento parece no ser muy alta. Aun así, el proceso de generación de las fuerzas en sentido vertical requiere mayor estudio ya que si el máximo valor de las fuerzas horizontales no ocurre de manera simultánea con el de las verticales, los métodos de cálculo han de sufrir un cambio importante. En segundo lugar, es relevante señalar que la correcta aplicación de una u otra metodología es fundamental para el adecuado diseño de las estructuras aquí estudiadas. Por lo que la estimación, compresión y justificación de los valores, parámetros, coeficientes y consideraciones son fundamentales en el éxito o fracaso de un proyecto de ingeniería.

\section{Agradecimiento}

Los autores desean expresar un amplio agradecimiento al Dr. Francisco Luis Martín Gallego por recomendaciones que enriquecieron de manera invaluable los contenidos y presentación del artículo.

\section{Referencias}

Bradbury, A.P.N, Allsop, N.H.W and Stevens, R.V. (1988) Hydraulic performance of breakwater crown walls. Hydraulic Research, Wallingford, Report SR146

Camus, P.B. and Guillén, J.G. (2004). Wave forces on crown walls: Evaluation of existing empirical formulations. Proceedings of $29^{\text {th }}$ Conference on Coastal Engineering, Lisboa, Portugal.

Günbak, A. R. and Gökce, T. (1984). Wave screen stability of rubble-mound breakwaters. International Symposium of Maritime Structures in the Mediterranean Sea. Athens, Greece.

Iribarren, R. y Nogales, C. (1964). Obras maritimas. Ed. Dossat, Madrid

Jensen, O. (1984) A monograph on rubble mound breakwaters. Danish Hydraulic Institute. Denmark

Li, X., Ren, B., Wang, G.Y. and Wang, Y. (2011). Numerical simulation of hydrodynamic characteristics on an arc crown wall using volume of fluid method based on BFC. Journal of Hydrodynamics 23(6), 767-776

Losada, M.A. and Giménez-Curto, L.A. (1981). Flow characteristics on rough, permeable slopes under wave action. Coastal Engineering 4, 187-206

Martin, F.L., Losada, M.A. and Medina, R. (1999). Wave loads on rubble mound breakwater crown walls. Coastal Engineering 37(2), 149-174

Martin, F.L., Vidal, C., Losada, I. and Martínez, C.L. (2000). Scale effects on scale tests on crown walls. Proceedings of $27^{\text {th }}$ International Conference on Coastal Engineering (ICCE), Ed. Billy Edge (ASCE), Sydney, Australia, 1696-1710 
Nagai, S. (1973). Wave forces on structures. Advances in Hydroscience, Vol. 9. Academic Press, New York, 253-324

Pedersen, J. (1996). Experimental study of wave forces and overtopping on crown walls of rubble mound breakwaters. Series paper 12, HC Engineering Laboratory, Department of Civil Engineering, Aalborg University, Denmark

Pedersen, J. and Burcharth, H.F. (1992). Wave forces on crown walls. Proceedings of $23^{\text {th }}$ Conference on Coastal Engineering, Venecia, Italia, 1489-1502
Takahashi, S., Kotake, Y., Fujiwra, R. and Isobe, M. (2002). Performance evaluation of perforated-wall caissons by VOF numerical simulations. Proceedings of the $28^{\text {th }}$ Coastal Engineering Conference, Cardiff, 1364-1376

Tørum, A., Moghim, M.N., Westeng, K., Hidayati, N. and Arntsen, Ø. (2012). On berm breakwaters: Recession, crown wall wave forces, reliability. Coastal Engineering 60, 299-318 